\title{
Arrtỉigo Origininal \\ Simpatectomia torácica por videotoracoscopia para tratamento da hiperidrose primária: estudo retrospectivo de 521 casos comparando diferentes níveis de ablação*
}

\author{
Video-assisted thoracic sympathectomy in the treatment of primary hyperhidrosis: \\ a retrospective study of 521 cases comparing different levels of ablation \\ Jorge Montessi ${ }^{1}$, Edmílton Pereira de Almeida ${ }^{2}$, João Paulo Vieira ${ }^{3}$, Marcus da Matta Abreu ${ }^{4}$, \\ Renato Lucas Passos de Souza ${ }^{5}$, Oswaldo Victor Duarte Montessi ${ }^{6}$
}

\begin{abstract}
Resumo
Objetivo: Comparar grau de satisfação dos pacientes simpatectomizados e presença de sudorese reflexa, de acordo com diferentes níveis de ablação. Métodos: Estudo retrospectivo de 521 pacientes com hiperidrose primária, submetidos à simpatectomia torácica no Hospital Monte Sinai e Hospital Universitário da Universidade Federal de Juiz de Fora - UFJF, de janeiro de 2001 a dezembro de 2005. Grupo I ( $\mathrm{n}=162$ ): termoablação do tronco simpático, tendo T2 como nível mais cranial da ressecção, independentemente de outros níveis seccionados caudalmente. Grupo II ( $n=65)$ : termoablação do tronco simpático, tendo T3 como nível mais alto. Grupo III ( $\mathrm{n}=294)$ : termoablação do tronco simpático, tendo T4 como nível mais alto. Resultados: Pós-operatório ótimo no controle da hiperidrose palmar/axilar em, respectivamente, 94/82\% dos pacientes do grupo I, 89/89\% do grupo II e 80/80\% do grupo III. Sudorese reflexa em 67\% dos pacientes dos grupos I e II, caindo para 61,29\% no grupo III devido à maior termoablação a partir de T4. Ocorreu sudorese reflexa severa em 32\% dos pacientes do grupo I, 9\% do grupo II e 4\% do grupo III. Conclusão: A simpatectomia propiciou excelente grau de satisfação e baixo índice de complicações. Não houve diferença na incidência de sudorese reflexa com diferentes níveis seccionados; porém, a intensidade desta complicação se mostrou menor quando optamos por níveis de bloqueio mais baixos, principalmente T4.
\end{abstract}

Descritores: Simpatectomia; Toracoscopia; Hiperidrose.

\begin{abstract}
Objective: To compare different levels of ablation in terms of the degree of patient satisfaction and extent of postoperative reflex sweating in sympathectomized patients. Methods: A retrospective study involving 521 patients with primary hyperhidrosis, submitted to thoracic sympathectomy at the Monte Sinai Hospital and University Hospital of the Federal University of Juiz de Fora, from January of 2001 to December 2005. All patients were submitted to thermal ablation of the sympathetic stem and were divided into three groups: up to T2 (group I, $n=162$ ); up to T3 (group II, $n=65$ ); and up to T4 (group III, $n=294$ ). Results: Optimal postoperative control of palmar/axillary hyperhidrosis was achieved in, respectively, 94/82\% of the patients of group I, 89/89\% of those in group II and 80/80\% of those in group III. Postoperative reflex sweating was observed in 67\% of the patients in groups I and II, compared with 61.29\% of those in group III. Severe reflex sweating occurred in 32\% of the group I patients, $9 \%$ of the group II patients and 4\% of the group III patients. Conclusion: Sympathectomy provided excellent patient satisfaction and a low incidence of complications. There was no significant difference between the levels of ablation in terms of reflex sweating, although the intensity of this complication decreased when lower levels of blockage, principally at the T4 level, were employed.
\end{abstract}

Keywords: Sympathectomy; Thoracoscopy; Hyperhidrosis.

\footnotetext{
* Trabalho realizado no Serviço de Cirurgia Torácica do Hospital Monte Sinai e Hospital Universitário da Universidade Federal de Juiz de Fora - UFJF - Juiz de Fora (MG) Brasil.

1. Doutor em medicina na área de Cirurgia Torácica. Universidade Federal de Juiz de Fora - UFJF - Juiz de Fora (MG) Brasil.

2. Titular da Sociedade Brasileira de Cirurgia Torácica. Universidade Federal de Juiz de Fora - UFJF - Juiz de Fora (MG) Brasil.

3. Titular da Sociedade Brasileira de Cirurgia Torácica. Faculdade de Ciências Médicas e da Saúde de Juiz de Fora - SUPREMA - Juiz de Fora (MG) Brasil.

4. Residente de Cirurgia Torácica. Universidade Federal de Juiz de Fora - UFJF - Juiz de Fora (MG) Brasil.

5. Acadêmico do $5^{\circ}$ ano de Medicina da Universidade Federal de Juiz de Fora - UFJF - Juiz de Fora (MG) Brasil.

6. Acadêmico do $3^{\circ}$ ano da Faculdade de Ciências Médicas e da Saúde de Juiz de Fora - SUPREMA - Juiz de Fora (MG) Brasil.

Endereço para correspondência: Jorge Montessi. Centro de Estudos Hospital Monte Sinai, Rua Vicente Beguelli, 315, Dom Bosco, CEP 36035-550, Juiz de Fora, MG, Brasil.

Tel 5532 3239-4194. Fax 5532 3239-4500. E-mail: jmontessi@terra.com.br

Recebido para publicação em 2/5/2006. Aprovado, após revisão, em 4/9/2006.
} 


\section{Introdução}

Hiperidrose primária ou essencial é um distúrbio caracterizado por sudorese excessiva e incontrolável, na ausência de causa discernível. ${ }^{(1)}$ É uma doença comum e pouco compreendida, freqüentemente exacerbada por estresse emocional ou fatores psicológicos. A hiperidrose se localiza preferencialmente nas axilas, palmas das mãos, plantas dos pés e face. Estima-se que afete de 0,6 a 1,0\% da população, geralmente adultos jovens. ${ }^{(1)} \mathrm{A}$ história familiar é positiva em 30 a $50 \%$ dos pacientes. ${ }^{(1)} \mathrm{A}$ hiperidrose focal localizada, de moderada a severa, está associada a considerável morbidade, incluindo maceração da pele, infecções cutâneas bacterianas e fúngicas secundárias. No entanto, a característica desta doença é o intenso desconforto do paciente, comprometendo sua vida social, afetiva e profissional. A hiperidrose primária, em geral, se manifesta no início da adolescência ou até mesmo na infância, não ocorre durante o sono, e pode ser agravada por estímulos emocionais. ${ }^{(1)}$ Há evidências de que a hiperidrose palmar primária é um distúrbio hereditário. ${ }^{(2)}$

0 suor excessivo nas palmas das mãos é a situação que mais se torna inconveniente em encontros profissionais e sociais. A hiperidrose axilar é uma condição comum e angustiante. A difícil aceitação social, o desconforto da umidade constante, o odor e o fato de manchar roupas diminuem significamente a qualidade de vida. ${ }^{(3)}$

0 tratamento clínico pode ser tópico, elétrico ou sistêmico. Em casos moderados de hiperidrose localizada, o uso de talcos absortivos e cloreto de alumínio a $20 \%$ pode ser suficiente. A iontoforese, ${ }^{(4)}$ quando bem administrada, é um método simples, algumas vezes efetivo, seguro e de custo razoável, no tratamento da hiperidrose essencial, de leve a moderada. 0 uso de medicação sistêmica também pode ser indicado; porém, com eficácia duvidosa e alta taxa de efeitos colaterais. Outra opção é o uso da toxina botulínica tipo A. Esta apresenta inúmeras vantagens; porém, o custo elevado para um efeito temporário dificulta o acesso da maioria dos pacientes. ${ }^{(5)}$

$\mathrm{Na}$ maioria dos casos, por não haver tratamentos capazes de resolver satisfatoriamente essa condição, os procedimentos cirúrgicos tornam-se necessários. Uma opção de tratamento baseia-se na remoção das glândulas écrinas e apócrinas da região axilar. Várias técnicas foram propostas, todas com alto índice de complicações..$^{(6-8)}$ A lipoaspiração das glândulas sudoríparas é outro método; porém, complicações como cicatrizes inestéticas, sangramento, recuperação demorada, necrose tecidual e infecção, limitam a sua indicação. ${ }^{(9)}$

A eficácia reduzida, a fugacidade do benefício e o elevado custo de outros métodos tópicos de tratamento da hiperidrose localizada e, muito especialmente, a disponibilidade crescente da cirurgia torácica vídeo-assistida, contribuíram decisivamente para a atual afirmação da simpatectomia como padrão-ouro no tratamento definitivo dos casos graves de hiperidrose palmar e axilar, onde a efetividade alcançada está acima de $98 \%,{ }^{(10)} \mathrm{com}$ elevado índice de satisfação dos pacientes. Em um estudo datado de 1951, foi realizada a primeira simpatectomia por toracoscopia. ${ }^{(11)}$ A partir de então, diferentes níveis do tronco simpático foram abordados no tratamento da sudorese excessiva. A evolução das técnicas videotoracoscópicas permitiu que a simpatectomia torácica pudesse ser realizada de forma bastante segura, possibilitando bons resultados e mínima morbidade.

As complicações relacionadas à manipulação da cavidade pleural, como hemotórax, pneumotórax, infecção de parede e edema de descompressão pulmonar, têm sido esporadicamente descritas, mas com uma incidência sempre inferior a $1 \% .{ }^{(10)}$ Algumas dificuldades, como as provocadas por aderências pleurais e a necessidade de re-simpatectomia (menos de 3\%) atribuíveis a alguma variante anatômica, são relatadas. ${ }^{(10)}$ A principal complicação da simpatectomia, sem dúvida, é a sudorese reflexa, sendo a mais comum e presente em mais de $50 \%$ dos pacientes, na maioria das séries. ${ }^{(12-18)}$

\section{Método}

Durante o período compreendido entre janeiro de 2001 e dezembro de 2005, 521 pacientes com hiperidrose primária foram submetidos à simpatectomia por videotoracoscopia no hospital Monte Sinai e Hospital Universitário da Universidade Federal de Juiz de Fora. Suas idades variaram de 9 a 69 anos. Considerando a distribuição de acordo com o sexo, os casos femininos predominaram, com 336 mulheres $(64,49 \%)$. A faixa etária predominante foi a de 20 a 29 anos, com 267 pacientes $(51,24 \%)$, seguida da de 10 a 19 anos com 132 pacientes (25,33\%). A 
manifestação clínica com maior incidência foi a hiperidrose palmo-plantar-axilar (34,7\%), seguida da palmo-plantar $(28,4 \%)$ e axilar (19\%). Quanto aos níveis de ablação, dividimos os pacientes em três grupos, considerando-se o nível inicial (cranial) de ablação (T2, T3 ou T4), independente dos outros níveis seccionados caudalmente: grupo I, constituído de pacientes com termoablação do tronco simpático a partir do segundo gânglio (T2); grupo II, constituído de pacientes com termoablação do tronco simpático a partir de T3; e grupo III, constituído de pacientes com termoablação do tronco simpático a partir de T4. Denominamos de termoablação de T2 a simpaticotomia realizada sobre o segundo arco costal, e assim sucessivamente. Na nossa série, não vimos a necessidade de realizar o isolamento cranial e caudal dos gânglios. A evolução clínica foi obtida através de controle ambulatorial e telefonemas. Foram formuladas questões simples, relacionadas ao desaparecimento dos sintomas, satisfação com o resultado final da cirurgia, e a presença e intensidade do suor reflexo. Os dados foram introduzidos em um programa criado para leitura no Sistema de Análise Estatística. As análises estatísticas foram feitas pelo teste qui-quadrado, considerando-se estatisticamente significativas as diferenças com $\mathrm{p}<0,05$.

\section{Resultados}

0 total de pacientes submetidos à simpatectomia torácica por videotoracoscopia em nosso seviço foi de $162(31,09 \%)$ no grupo I, 65 (12,47\%) no grupo II, e $294(56,42 \%)$ no grupo III. Nos grupos I e III, 63\% dos pacientes eram do sexo feminino, enquanto que no grupo II, $62 \%$. A hiperidrose palmar foi queixa de $90 \%$ dos pacientes do grupo I, 70,5\% do grupo II e 61\% do grupo III. A hiperidrose axilar foi relatada por 59,5\% dos pacientes do grupo I, por 72,3\% do grupo II e por $77,4 \%$ do grupo III.

Inicialmente, empregávamos as ablações de T2 e T3 para o tratamento das hiperidroses palmar e axilar. Após relevantes publicações, ${ }^{(19)}$ passamos a realizar a ablação a partir de T4, e pudemos perceber a redução na incidência de hiperidrose reflexa, a qual diminuiu de 67\% nos grupos I e II para 61,29\% no grupo III $(p<0,05)$. (Figura 1)

A sudorese reflexa considerada severa ocorreu em 32\% dos pacientes do grupo I, 9\% do grupo II,

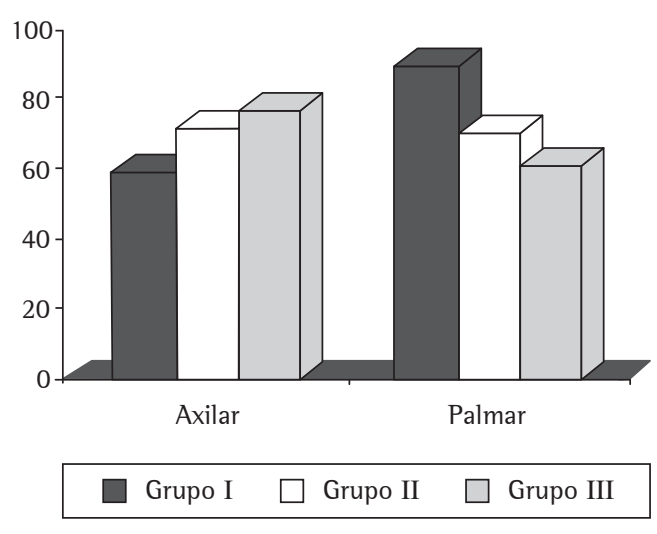

Figura 1 - Incidência em percentual de hiperidrose palmar e plantar de acordo com o grupo estudado: grupo I) simpatectomia a nível de T2; grupo II) simpatectomia a nível de T3; e grupo III) simpatectomia a nível de T4.

e $4 \%$ do grupo III $(p<0,05)$. A forma moderada ocorreu em 18\% dos pacientes do grupo I, em $31 \%$ dos pacientes do grupo II, e 36\% no grupo III. Já a forma leve ocorreu em 50\% no grupo I e 60\% nos grupos II e III. Estas categorias foram apresentadas aos pacientes que relataram qualquer grau de hiperidrose reflexa, quando thes pedimos que caracterizassem seu quadro clínico. (Figura 2)

A incidência de complicações pós-operatórias foi de 3,07\%, dado este estatisticamente não significativo. Em 12 casos (2,3\%) ocorreu pneumotórax, sendo que a drenagem torácica em selo d'água foi

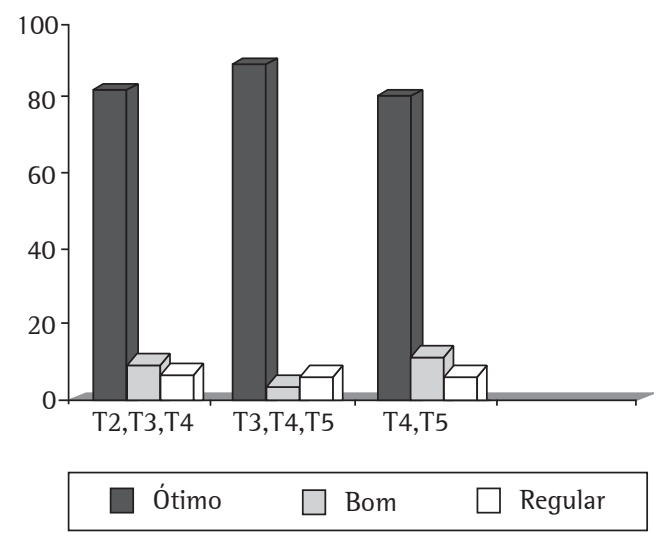

Figura 2 - Grau de satisfação da hiperidrose axilar de acordo com o nível de ablação: 1) Ótimo; 2) Bom; e 3) Regular. 
utilizada em 9 casos, e o dreno torácico permaneceu por um período inferior a $12 \mathrm{~h}$ em todos os casos; nos demais, optamos pela conduta conservadora. A síndrome de Horner transitória foi observada em 2 pacientes $(0,38 \%)$, que foram submetidos à ablação de T2; porém, a mesma reverteu-se em um período de uma semana após a cirurgia. Foi observada, também, a presença de derrame pleural como complicação em 2 casos (0,38\%), sendo que a toracocentese foi suficiente para a resolução do problema.

0 nosso método obteve falha unilateral em 6 casos $(1,15 \%)$, sendo 4 pacientes re-operados. Nova cirurgia foi recusada por 2 pacientes.

0 grau de satisfação foi considerado ótimo por $85 \%$ dos pacientes, bom por $12 \%$ e regular ou ruim em 3\% dos casos. Esta classificação quanto à satisfação com o procedimento foi obtida espontaneamente durante entrevista clínica com os pacientes e, quando isto não ocorria, oferecíamos aos pacientes quatro opções de escolha para que pudessem classificar o resultado final da cirurgia. Houve arrependimento em apenas 1\% dos pacientes.

Com relação ao grau de satisfação por território, 94\% dos pacientes do grupo I, com hiperidrose palmar, consideraram o resultado da simpatectomia ótimo, e 3\% consideraram o resultado bom. Ótimo também foi considerado por $89 \%$ dos pacientes do grupo II, e bom por 6\%. Nos pacientes do grupo III, $80 \%$ consideraram o resultado ótimo, e 12\% bom. $\mathrm{Na}$ hiperidrose axilar, $82 \%$ dos pacientes do grupo I consideraram o resultado da simpatectomia ótimo e 10\% bom, sendo ótimo também em $89 \%$ dos pacientes do grupo II e bom em 5\%. Nos pacientes do grupo III, 80\% consideraram o resultado ótimo e $12 \%$ bom, dados estes estatisticamente significativos. (Figura 3 e 4 )

\section{Discussão}

A hiperidrose pode ser primária (idiopática ou essencial) ou secundária a uma variedade de doenças ou, eventualmente, ao uso de determinadas drogas, como antidepressivos tricíclicos e venlafaxina. Independente do tipo ou da causa, é uma condição socialmente embaraçante e funcionalmente incapacitante. ${ }^{(20)}$

A eficácia da simpatectomia no controle da hiperidrose palmar e axilar é conhecida há muitas

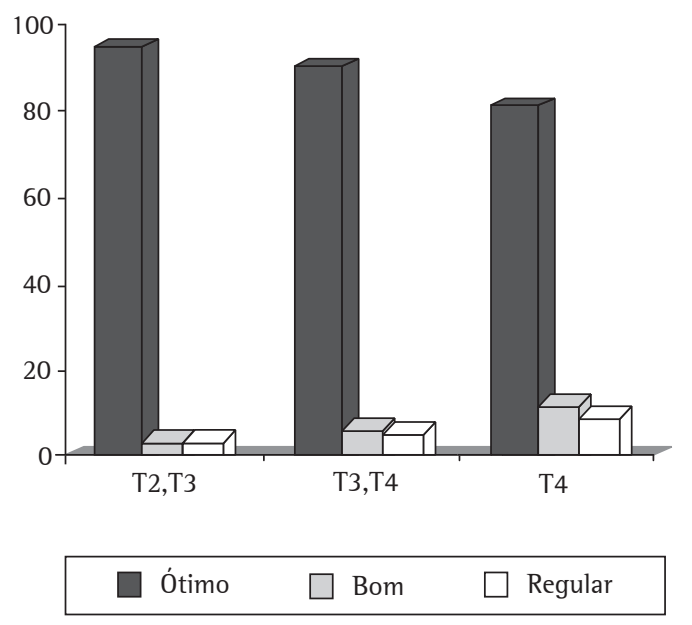

Figura 3 - Grau de satisfação da hiperidrose palmar de acordo com o nível de satisfação: 1) Ótimo; 2) Bom; e 3) Regular.

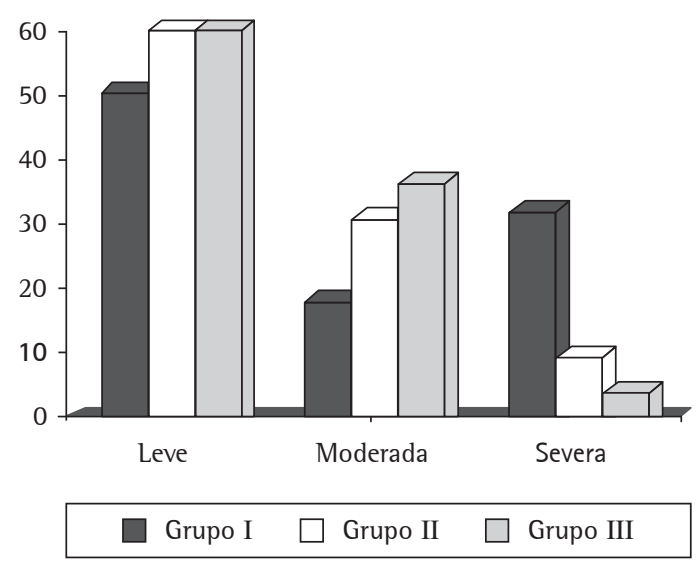

Figura 4 - Incidência de sudorese reflexa de acordo com os grupos estudados: grupo I) simpatectomia a nível de T2; grupo II) simpatectomia a nível de T3; e grupo III) simpatectomia a nível de T4.

décadas. A complexidade do procedimento, quando realizado por via cervical, ou a sua morbidade, quando associado a duas toracotomias axilares, fez com que seu uso, no passado, fosse restrito a casos muito selecionados.

A primeira simpatectomia para tratamento da hiperidrose foi realizada em $1920 .{ }^{(21)} \mathrm{A}$ via utilizada foi a cervical anterior. Em 1942, ${ }^{(22)}$ houve um grande avanço na técnica, ficando demonstrado não ser necessário o sacrifício do gânglio estrelado para 
denervar o membro superior. Esse fato é de grande relevância porque praticamente eliminou uma das complicações mais temidas da simpatectomia torácica, a síndrome de Claude-Bernard-Horner.

Outro autor, em 1949, ${ }^{(23)}$ descreveu a via axilar e, em 1954, relatou uma série de 26 pacientes operados, ressaltando as vantagens desta abordagem quando da realização de simpatectomia aberta. A abordagem minimamente invasiva do tronco simpático iniciouse em 1942, ${ }^{(15)}$ quando foi descrita a simpatectomia endoscópica, com anestesia local, em posição semisentada, com dois portais de entrada.

Em 1950, ${ }^{(11)}$ foi apresentada uma técnica em 500 pacientes, tornando o método mais popular, o qual, com algumas variações, é utilizado pela maioria dos serviços atuais.

A era da videocirurgia iniciou-se na década de 80, quando foi realizada a primeira simpatectomia por videotoracoscopia. ${ }^{(24)} \mathrm{Em}$ 1996, foi feito um levantamento nos hospitais da república chinesa, publicando a experiência do país com 9988 pacientes operados. Esse fato produziu uma explosão mundial na realização da cirurgia. Ficou claro, com este levantamento, que o procedimento era exeqüível em posição semi-sentada, permitindo facilmente o procedimento bilateral, não sendo necessária a utilização de intubação seletiva e, nem mesmo, na maioria dos casos, a insuflação de gás carbônico na cavidade pleural. 0 autor relatou, também, um baixo índice de complicações imediatas e alta taxa de sucesso. ${ }^{(24)}$ Ficou evidente, todavia, a alta prevalência de hiperidrose reflexa, quando se faz ablação de T2 e T3.(24)

A sudorese reflexa é a complicação mais comum pós simpatectomia, ocorrendo, geralmente, no tronco e membros inferiores, sendo rara a sudorese reflexa plantar. Esta complicação é responsável pelos casos de insatisfação com a cirurgia, motivando grande interesse dos cirurgiões em descobrir meios para diminuir ou eliminar a ocorrência deste evento indesejável. ${ }^{(14,17,24)}$ Por muito tempo, pensou-se que a sudorese reflexa se relacionava com o número de estações nodais ressecadas, motivando vários autores a realizarem simpatectomias em um único nível ganglionar. ${ }^{(12,17,24)}$ Em levantamento de quase dez mil pacientes simpatectomizados em nível T2, não foi mostrada uma redução da prevalência da mesma. ${ }^{(24)}$

Nesse contexto, surgiu um trabalho na Holanda, constituído por uma série de 14 pacientes com ablação de T3 para tratamento da hiperidrose palmar, com sucesso equivalente à ablação T2-T3; porém, com ausência de hiperidrose reflexa. ${ }^{(17)}$

De grande relevância foi um estudo randomizado envolvendo 171 pacientes, comparando a simpatectomia em T3-T4, T4, e T4-T5 para tratamento da hiperidrose e bromidrose axilar. Relatou-se uma redução de $70 \%$ de hiperidrose reflexa, no grupo T3-T4, para 29\% nos grupos T4 e T4-T5, demonstrando-se a importância de T3 na prevalência da sudorese reflexa. Ficou evidente, também, para tratamento da hiperidrose axilar, a necessidade de ablação de T4 e T5. ${ }^{(14)}$

Mesmo com a evolução da técnica, o inconveniente da simpatectomia continuava sendo a presença, em número significativo de pacientes, da sudorese reflexa, apesar de apenas uma minoria dos pacientes ficarem insatisfeitos com a cirurgia. ${ }^{(12-15)}$ Surgiu, então, a idéia da clipagem do tronco simpático, em substituição à termoablação, com a intenção de reversão da cirurgia, caso o paciente se apresentasse insatisfeito com o resultado final do procedimento. Porém, o número de reversões é muito baixo na maioria das séries. Hoje, sabe-se, também, que esta reversão deve ocorrer dentro de um prazo de aproximadamente 30 dias após a cirurgia, pois, após este período, o sucesso da reversão ocorre em apenas aproximadamente $25 \%$ dos pacientes. ${ }^{(25)}$

0 grande mérito da clipagem foi uma descoberta acidental. ${ }^{(26)}$ Numa experiência de 165 pacientes submetidos à clipagem do tronco simpático, 1 paciente apresentou sudorese reflexa compensatória severa de um só lado. 0 controle radiológico revelou que a mesma ocorreu apenas no lado em que o clipe tinha sido colocado na posição correta, ou seja, em T2. No outro lado, em que o clipe tinha sido inadvertidamente colocado mais abaixo (T3), não houve sudorese reflexa. 0 controle da hiperidrose palmar, entretanto, tinha sido igualmente alcançado nos dois lados. A partir de então, esses autores operaram 100 pacientes consecutivos com secção da cadeia ao nível de T3, e não encontraram mais sudorese reflexa severa. No seguimento da observação, passaram a amputar a cadeia ao nível de T4 e a sudorese reflexa revelou-se ainda mais leve; entretanto, em alguns poucos casos, com a secção ao nível de T4, houve falha no controle da hiperidrose palmar, e alguns pacientes precisaram ser re-operados para uma nova secção da cadeia em um nível mais cranial. Constataram que, nos casos 
de simpatectomia lombar bilateral, não há relato de sudorese reflexa, e sugeriram como explicação para este fenômeno a observação de que o sistema nervoso autônomo age por mecanismos de feedback hipotalâmico positivo ou negativo. Mais surpreendente foi a demonstração de que a ablação de T4 controla a hiperidrose palmar; e a ablação de T4-T5, a hiperidrose palmar, axilar e a bromidrose. ${ }^{(19)}$

Em nosso estudo, passamos a realizar a ablação T4-T5 para controle da forma axilar em razão de relevantes estudos, principalmente um publicado em 2001. ${ }^{(19)}$ Antes disso, a incidência de sudorese reflexa era de $67 \%$ entre os pacientes de nossa casuística, sendo, atualmente, de 61,29\%. A sudorese reflexa severa na termoablação de T2 ocorreu em 32\% dos casos. Esta incidência reduziu-se para 6\% nos pacientes sem termoablação de T2.

A presença de sudorese reflexa e a falha do método estão em intima relação com o grau de satisfação pós-operatória dos pacientes. Uma rápida revisão bibliográfica acerca da sudorese reflexa resulta no encontro de números díspares, onde há casuísticas em que a prevalência situa-se em torno de 5\%, ao mesmo tempo em que outras publicações, igualmente respeitáveis, reportam incidência na faixa de $85 \% .{ }^{(27,28)}$ Fica claro que variações dessa dimensão têm como significado a falta de clara definição do quadro, já demonstrada por diversos autores. ${ }^{(28,29)}$

Vários autores, utilizando métodos sofisticados de quantificação do suor reflexo, como gravimetria e sudometria, encontraram importantes discrepâncias entre o desconforto relatado e o suor efetivamente quantificado. ${ }^{(27,30)} \mathrm{A}$ intensidade da sudorese reflexa guarda estreita relação com a temperatura ambiente, umidade e atividade física, sendo pior tolerada em climas úmidos e quentes. Além disso, um mesmo paciente pode quantificar de maneira bastante diferente a intensidade da sudorese num intervalo de poucos dias. Em nosso estudo, procuramos adotar um método frequentemente encontrado na literatura, semiquantitativo, classificando o suor reflexo em leve, moderado ou intenso, levando em conta a impressão do paciente.

A simpatectomia bilateral por videotoracoscopia constitui uma intervenção simples e de baixa morbidade para o tratamento definitivo da hiperidrose primária craniofacial, palmar e axilar.

A sudorese reflexa é o mais importante efeito colateral da simpatectomia, sendo usualmente bem tolerado, e pouco interfere no resultado cirúrgico. Em alguns casos, contudo, pode tornar-se complicação séria e, por isso, motivo de arrependimento por parte dos pacientes.

0 grande avanço que houve recentemente foi a identificação do segundo gânglio simpático como provável centro da sudorese reflexa, e sua preservação é a maneira mais segura de prevenir a ocorrência da mesma, sem comprometer o controle efetivo da hiperidrose palmar e axilar. Preservado o segundo gânglio, pode-se, ainda, observar algum grau de sudorese reflexa; porém, geralmente leve, tolerável e fugaz. Desta forma, modificações no nível da simpatectomia têm sido consideradas, principalmente evitando a termoablação de T2.

Após revisão da literatura, optamos por termoablação de T3 para o controle da hiperidrose craniofacial, T4 para os casos de queixa palmar e T4-T5 para a hiperidrose axilar.

\section{Referências}

1. Stolman LP. Treatment of hyperhidrosis. Dermatol Clin. 1998;16(4):863-9.

2. Ro KM, Cantor RM, lange KL, Ahn SS. Palmar hyperhidrosis: evidence of genetic transmission. J Vasc Surg. 2002;35(2):382-6.

3. Amir M, Arish A, Weinstein Y, Pfeffer M, Levy Y. Impairment in quality of life among patients seeking surgery for hyperhidrosis (excessive sweating): preliminary results. Isr J Psychiatry Relat Sci. 2000;37(1):25-31.

4. Holzle E, Alberti N. Long-term efficacy and side effects of tap water iontophoresis of palmoplantar hyperhidrosis-the usefulness of home therapy. Dermatologica. 1987;175(3):126-35.

5. Hexsel AM, Hexsel CL. Toxina Botulínica no Tratamento das Hiperidroses. In: Almeida ART, Hexsel DM, editors. Hiperidrose e Toxina Botulínica. São Paulo: Know-how Editorial; 2003. p. 145-227.

6. Skoog T, Thyresson N. Hyperhidrosis of the axillae. A method of surgical treatment. Acta Chir Scand. 1962;(124):531-8.

7. Sabatier H, Picaud AJ. The surgical treatment of axillary hyperhidrosis. J Dermatol Surg. 1976;2(4):331-2.

8. Eldh J, Fogdestam 1. Surgical treatment of Hyperhidrosis axillae. Scand J Plast Reconstr Surg. 1976;10(3):227-9.

9. Swinehart JM. Treatment of axillary hyperhidrosis: combination of the starch-iodine test with the tumescent liposuction technique. Dermatol Surg. 2000;26(4):392-6.

10. Chung $\mathrm{HH}$, Oh CS, Koh KS, Kim HJ, Paik HC, Lee DY. Anatomic variations of the T2 nerve root (including the nerve of Kuntz) and their implications for sympathectomy. J Thorac Cardiovasc Surg. 2002;123(3):498-501.

11. Kux E. The endoscopic approach to the vegetative nervous system and its therapeutic possibilities; especially in duodenal ulcer, angina pectoris, hypertension and diabetes. Dis Chest. $1951 ; 20(2): 139-47$. 
12. Drott C, Gothberg G, Claes G. Endoscopic procedures of the upper-thoracic sympathetic chain. A review. Arch Surg. 1993;128(2):237-41.

13. Gossot D, Toledo L, Fritsch S, Célérier M. Thoracoscopic sympathectomy for upper limb hyperhidrosis: Looking for the right operation. Ann Thorac Surg. 1997;64(4):975-8.

14. Hsu CP, Shia SE, Hsia JY, Chuang CY, Chen CY. Experiences in thoracoscopic sympathectomy for axillary hyperhidrosis and osmidrosis: focusing on the extent of sympathectomy. Arch Surg. 2001;136(10):1117-8.

15. Kux M. Thoracic Endoscopic sympathectomy in palmar and axillary hyperhidrosis. Arch Surg. 1978;113(3):264-6.

16. Reisfeld R, Nguyen R, Pnini A. Endoscopic thoracic sympathectomy for hyperhidrosis: Experience with both cauterization and clamping methods. Surg Laparosc Endosc Percutan Tech. 2002;12(4):255-7.

17. Riet MV, Smet AA, Kuiken H, Kazemier G, Bonjer HJ. Prevention of compensatory hyperhidrosis after thoracoscopic sympathectomy for hyperhidrosis. Surg Endosc. 2001;15(10):1159-62.

18. Zacher J, Imhof M, Huber ER, Plas EG, Herbst F, Jaskesz $\mathrm{R}$, et al. Video assistance reduces complication rate of thoracoscopic sympathicotomy for hyperhidrosis. Ann Thorac Surg. 1999;68(4):1177-81.

19. Lin CC, Telaranta T. Lin-Telaranta classification: the importance of different procedures for different indications in sympathetic surgery. Ann Chir et Gynaecol. 2001;90(3):161-6

20. Almeida EP, Montessi J, Vieira JP, Marsico GA. Simpatectomia Torácica. HU Revista. 2004;30(1):23-6.

21. Mack M. Thoracoscopy. In: Pearson FG, Cooper JD, Deslauriers J, Ginsberg RJ, Hiebert C, Patterson GA, Urschel HC, editors. Thoracic Surgery. 1st ed. New York: Churchill Livingstone; 1995. p. 1488-509.
22. Hyndman OR, Wolking J. Sympathectomy of the upper extremity: evidence that only the second dorsal ganglion need be removed for complete sympathectomy. Arch Surg. 1942;(45):145-55.

23. Atkins HJ. Sympathectomy by the axillary approach. Lancet. 1954;266(6811):538-9.

24. Kao MC, Lin JY, Chen YL, Hsieh CS, Cheng LCJ, Huang SJ. Minimally invasive surgery: video endoscopic thoracic sympathectomy for palmar hyperhidrosis. Ann Acad Med Singapore. 1996;25(5):673-8.

25. Lin TS. Endoscopic clipping in video-assisted thoracoscopic sympathetic blockade for axillary hyperhidrosis. An analysis of 26 cases. Surg Endosc. 2001;15(2):126-8.

26. Lin $\mathrm{CC}, \mathrm{Wu} \mathrm{HH}$. Endoscopic t4-sympathetic block by clamping (ESB4) in treatment of hyperhidrosis palmaris et axillaris--experiences of 165 cases. Ann Chir Gynaecol. 2001;90(3):167-9.

27. Gossot D, Kabiri H, Caliandro R, Debrosse D, Girard P, Grunenwald D. Early complications of thoracic endoscopic sympathectomy: a prospective study of 940 procedures. Ann Thorac Surg. 2001;71(4):116-9.

28. Leão LEV, Oliveira R, Szule R, Mari JJ, Crotti PL, Gonçalves JJ. Role of video-assisted thoracoscopic sympathectomy in the treatment of primary hyperhidrosis. São Paulo Med J. 2003;121(5):191-7.

29. Gossot D, Galetta D, Pascal A, Debrosse D, Caliandro R, Girard $\mathrm{P}$, et al. Long-term results of endoscopic thoracic sympathectomy for upper limb hyperhidrosis. Ann Thorac Surg. 2003;75(4):1075-9.

30. Hashmonai M, Kopelman D, Assalia A. The treatment of primary palmar hyperhidrosis: a review. Surg Today. 2000;30(3):211-8. 